\title{
2-Type flat integral submanifolds in $S^{7}(1)$
}

\author{
Christos Baikoussis ${ }^{1}$ and David E. BlaiR
}

(Received October 3, 1994; Revised December 13, 1994)

\begin{abstract}
This paper determines all flat, mass-symmetric, 3-dimensional 2-type submanifolds of the unit sphere $S^{7}(1)$ which are integral submaniifolds of the canonical contact structure.
\end{abstract}

Key words: integral submanifolds, finite type submanifolds.

\section{Introduction}

In $[5,6]$ Bang-Yen Chen introduced the notion of submanifolds of finite type. Let $M$ be a submanifold of Euclidean space $E^{n}$ and $\Delta$ the Laplacian of the induced metric. $M$ is said to be of finite type if its position vector field $x$ has a decomposition of the form

$$
x=x_{0}+x_{1}+\cdots+x_{k}
$$

where $x_{0}$ is a constant vector and $\Delta x_{i}=\lambda_{i} x_{i}$. Assuming the $\lambda_{i}$ to be distinct we say that $M$ is of $k$-type.

The theory of finite type submanifolds has become an area of active research. The first results on this subject have been collected in the book [6]; for a recent survey, see [7]. In particular, there is the problem of classification of low type submanifolds which lie in a hypersphere. Far from being solved in general, there are many partial results which contribute to the solution of this problem. For instance, by the well-known result of Takahashi [10], 1-type submanifolds are characterized as being minimal in a sphere.

However, classification of even 2-type spherical submanifolds seems to be virtually impossible. A compact submanifold $M^{n}$ of a hypersphere $S^{m}$ of $E^{m+1}$ is said to be mass-symmetric if the center of mass of $M^{n}$ in $E^{m+1}$ is the center of $S^{m}$ in $E^{m+1}$. Note that the only 2-type surface in $S^{3}$ is the flat torus $S^{1}(a) \times S^{1}(b), a \neq b$, while a 2-type mass-symmetric integral surface in $S^{5}$ is locally the product of a circle and a helix of order 4 , or

\footnotetext{
${ }^{1}$ This work was done while the first author was a visiting scholar at Michigan State University.

1991 Mathematics Subject Classification : 53C25, 53C40, 53C15.
} 
the product of two circles [1]. Integral finite type surfaces in $S^{5}$ are also studied in [3], where a weaker assumption is used. On the other hand the codimension seems to play a crucial role in the characterization of low type spherical submanifolds of codimension greater than 1 and hence it seems to be necessary to use extra conditions. In [7] B.-Y. Chen gives a list of open problems and conjectures concerning submanifolds of finite type. $\mathrm{He}$ also gives a good survey of what is known about the classification of 2-type (spherical) submanifolds with arbitrary codimension.

This paper provides a contribution in codimension greater than 1 by classifying 2-type, mass-symmetric, flat integral 3-dimensional submanifolds of the unit sphere $S^{7}(1) \subset E^{8}$.

It is well-known [4] that an odd-dimensional sphere $S^{2 n+1}$ carries a contact structure, i.e., a global 1-form $\eta$ such that $\eta \wedge(d \eta)^{n} \neq 0$. A submanifold of a contact manifold $M^{2 n+1}$ with contact form $\eta$ is an integral submanifold if it is an integral submanifold of the $2 n$-dimensional subbundle defined by $\eta=0$. It is well known that the maximum dimension of an integral submanifold is only $n$ and hence of dimension at most 3 in $S^{7}$. Moreover, contact transformations map integral submanifolds to integral submanifolds and hence integral submanifolds are fundamental objects in the geometry of contact manifolds. For a general discussion of these ideas see [4, Chap. III] or [9].

Theorem Let $x: M \rightarrow S^{7} \subset E^{8}$ be an isometric immersion of a flat 3dimensional mass-symmetric 2-type integral submanifold $M$ into $S^{7}$. Then $M$ lies fully in $S^{7} \subset E^{8} \cong \mathbb{C}^{4}$ and the position vector $x=x(u, v, w)$ of $M$ in $E^{8}$ is given by

$$
\begin{aligned}
x= & \frac{\lambda}{\sqrt{\lambda^{2}+1}} \cos \frac{u}{\lambda} e_{1}+\frac{1}{\sqrt{\sigma_{2}\left(\sigma_{1}+\sigma_{2}\right)}} \sin \left(\lambda u-\sigma_{2} v\right) e_{2} \\
& +\frac{1}{\sqrt{\rho_{1}\left(\rho_{1}+\rho_{2}\right)}} \sin \left(\lambda u+\sigma_{1} v+\rho_{1} w\right) e_{3} \\
& +\frac{1}{\sqrt{\rho_{2}\left(\rho_{1}+\rho_{2}\right)}} \sin \left(\lambda u+\sigma_{1} v-\rho_{2} w\right) e_{4} \\
& +\frac{\lambda}{\sqrt{\lambda^{2}+1}} \sin \frac{u}{\lambda} e_{5}+\frac{1}{\sqrt{\sigma_{2}\left(\sigma_{1}+\sigma_{2}\right)}} \cos \left(\lambda u-\sigma_{2} v\right) e_{6} \\
& +\frac{1}{\sqrt{\rho_{1}\left(\rho_{1}+\rho_{2}\right)}} \cos \left(\lambda u+\sigma_{1} v+\rho_{1} w\right) e_{7}
\end{aligned}
$$




$$
+\frac{1}{\sqrt{\rho_{2}\left(\rho_{1}+\rho_{2}\right)}} \cos \left(\lambda u+\sigma_{1} v-\rho_{2} w\right) e_{8}
$$

with $\rho_{1}=\frac{1}{2}\left(\sqrt{4 c(2 c-a)+d^{2}}+d\right), \rho_{2}=\frac{1}{2}\left(\sqrt{4 c(2 c-a)+d^{2}}-d\right), \sigma_{1}=$ $c, \sigma_{2}=c-a$, where $a, c, d, \lambda$ are constants such that $-1 \leq \lambda<0,1+\lambda^{2}+$ $a c-c^{2}=0, a \geq 0, a^{2} \geq d^{2}$ and $\left\{e_{1}, e_{2}, e_{3}, e_{4}, e_{5}=-J e_{1}, e_{6}=-J e_{2}\right.$, $\left.e_{7}=-J e_{3}, e_{8}=-J e_{4}\right\}$ is an orthonormal basis of $\mathbb{C}^{4}$.

\section{Preliminaries}

First we briefly describe the Sasakian structure on $S^{7}$. We consider the space $\mathbb{C}^{4}$ of 4 -complex variables. Let $J$ denote its natural complex structure, namely by identifying $z=\left(x_{1}+i y_{1}, \ldots, x_{4}+i y_{4}\right) \in \mathbb{C}^{4}$ with $\left(x_{1}, \ldots, x_{4}, y_{1}, \ldots, y_{4}\right) \in E^{8}, J z=\left(-y_{1}, \ldots,-y_{4}, x_{1}, \ldots, x_{4}\right)$. We give the unit sphere $S^{7}=\left\{z \in \mathbb{C}^{4}:|z|=1\right\}$ its usual contact structure. Define a tangent vector field $\xi$, a 1-form $\eta$ and a $(1,1)$ tensor field $\varphi$ on $S^{7}$ as follows:

Let $\langle$,$\rangle denote the metric on S^{7}$ induced from $\mathbb{C}^{4}$ (so $S^{7}$ has constant sectional curvature 1),

$$
\xi=-J z, \eta(X)=\langle X, \xi\rangle \text { and } \varphi=s \circ J
$$

where $s$ denotes the orthogonal projection from $T_{z} \mathbb{C}^{4}$ onto $T_{z} S^{7}$ and $X$ an arbitrary tangent vector field on $S^{7}$. Using these definitions, we obtain for all tangent vector fields $X$ and $Y$ on $S^{7}$ that

$$
\begin{aligned}
& \varphi^{2} X=-X+\eta(X) \xi, \\
& \eta(\xi)=1, \eta(X)=\langle X, \xi\rangle, d \eta(\xi, X)=0, \\
& d \eta(X, Y)=\langle X, \varphi Y\rangle, \\
& N=-2 d \eta \otimes \xi
\end{aligned}
$$

where $N$ is the Nijenhuis tensor of $\varphi$ given by $N(X, Y)=[\varphi X, \varphi Y]+$ $\varphi^{2}[X, Y]-\varphi[\varphi X, Y]-\varphi[X, \varphi Y]$. It is well-known [4] that these formulas imply that $(\varphi, \xi, \eta,\langle\rangle$,$) determines a Sasakian structure on S^{7}$. Therefore, we also have

$$
\nabla_{X}^{\prime} \xi=-\varphi X,\left(\nabla_{X}^{\prime} \varphi\right) Y=\langle X, Y\rangle \xi-\eta(Y) X
$$

where $\nabla^{\prime}$ denotes the Levi-Civita connection of $\langle$,$\rangle . For more details see$ [4]. 
A Riemannian manifold $M$, isometrically immersed in $S^{7}$, is called an integral submanifold if $\eta$ restructed to $M$ vanishes. Some authors call integral submanifolds, $C$-totally real submanifolds. A direct consequence of the definition is that $\varphi(T M) \subset T^{\perp} M$ (i.e., that $M$ is an anti-invariant submanifold of $S^{7}$ ), in particular, $d \eta=0$ on $M$.

In this paper we consider the unit hypersphere $S^{7}(1) \subset \mathbb{C}^{4} \cong E^{8}$ centered at the origin and with the Sasakian structure $(\varphi, \xi, \eta,\langle\rangle$,$) .$

Let

$$
x: M \rightarrow S^{7}(1)
$$

be an immersion of a 3-dimensional integral submanifold $M$ into $S^{7}(1)$. Denote by $\bar{\nabla}$ the usual Levi-Civita connection of $E^{8}$ and by $\nabla, \nabla^{\prime}$ the induced connections on $M$ and $S^{7}(1)$, respectively. Let $H, h, A$ and $D$ denote the mean curvature vector, the second fundamental form, the Weingarten maps and the normal connection of $M$ in $E^{8}$, respectively. Finally, denote by $H^{\prime}, h^{\prime}, A^{\prime}$ and $D^{\prime}$ the corresponding quantities for $M$ in $S^{7}(1)$. Then we have $H=H^{\prime}-x$ and, for any vector $\zeta$ normal to $M$ in $S^{7}(1), A_{\zeta}=A_{\zeta}^{\prime}$. If $X_{1}, X_{2}, X_{3}$ is a local orthonormal basis of vector fields on $M$, then $\xi_{i}=\varphi X_{i}, i=1,2,3, \xi_{0}=\xi, x$ form a basis of the normal space of $M$ in $E^{8}$. For convenience, we put $\left(e_{1}, \ldots, e_{8}\right)=\left(X_{1}, X_{2}, X_{3}, \xi_{1}, \xi_{2}, \xi_{3}, \xi_{0}, x\right)$, and denote by $\left\{w_{i}\right\}, i=1, \ldots, 8$ the dual frame of $\left\{e_{i}\right\}$ and by $\left\{w_{i}^{j}\right\}, i, j=1, \ldots, 8$ the corresponding connection forms. Thus we have $\bar{\nabla} e_{i}=\sum_{i=1}^{8} w_{i}^{j} e_{j}$. If $A_{s}$ is the Weingarten maps with respect to $\xi_{s}$, then from [4, pp. 102-103], we have $A_{i} X_{j}=A_{j} X_{i}, i, j=1,2,3$ and $A_{0}=0$. Thus, by means of straightforward calculation and using the Gauss-Weingarten formulas we obtain for the tangent vector fields $X_{i}$ on $M$

$$
\begin{aligned}
& \varphi h^{\prime}\left(X_{i}, X_{j}\right)=-A_{\varphi X_{j}} X_{i}, \\
& \left\langle h\left(X_{i}, X_{j}\right), \varphi X_{k}\right\rangle=\left\langle h\left(X_{i}, X_{k}\right), \varphi X_{j}\right\rangle .
\end{aligned}
$$

On the other hand, using the Gauss-Weingarten formulas, (2.2) and (2.4) we obtain $\sum_{\ell=1}^{8} w_{3+j}^{\ell}\left(e_{i}\right) e_{\ell}=\delta_{i j} e_{7}+\sum_{\ell=1}^{6} w_{j}^{\ell}\left(e_{i}\right) \varphi e_{\ell}, i, j=1,2,3$. Thus

$$
w_{3+i}^{3+j}=w_{i}^{j}, w_{3+j}^{7}\left(e_{i}\right)=\delta_{i j}, w_{3+j}^{8}=w_{7}^{8}=0, i, j=1,2,3 .
$$

The sectional curvature $K\left(X_{i}, X_{j}\right)$ of $M$ determined by an orthonormal pair $X_{i}, X_{j}$ is given by 


$$
K\left(X_{i}, X_{j}\right)=1+\sum_{\ell=1}^{3}\left(\left\langle A_{\ell} X_{i}, X_{i}\right\rangle\left\langle A_{\ell} X_{j}, X_{j}\right\rangle-\left\langle A_{\ell} X_{i}, X_{j}\right\rangle^{2}\right) .
$$

The covariant derivative $\bar{\nabla} h$ is defined by

$$
\begin{aligned}
\bar{\nabla} h\left(X_{i}, X_{j}, X_{k}\right)= & D_{X_{i}} h\left(X_{j}, X_{k}\right)-h\left(\nabla_{X_{i}} X_{j}, X_{k}\right) \\
& -h\left(X_{j}, \nabla_{X_{i}} X_{k}\right) .
\end{aligned}
$$

We say that the submanifold $M$ is $C$-parallel if the vector $\bar{\nabla} h$ is parallel to the characteristic vector field $\xi$.

\section{2-type Submanifolds}

Let $M$ be a 3 -dimensional integral submanifold of $S^{7}(1)$. In what follows we will always work with an orthonormal basis on a component of an open dense subset $U \subset M$ constructed in the following way. Let $p \in M$. Consider the function $f: U M_{p} \rightarrow \mathbb{R}: u \rightarrow f(u)=\langle h(u, u), \varphi u\rangle$, where $U M_{p}$ is the unit sphere in the tangent space $M_{p}$. Since $U M_{p}$ is compact, $f$ attains an absolute maximum at a unit vector $X_{1}$. We deduce from (2.4) that $\left\langle h\left(X_{1}, X_{1}\right), \varphi w\right\rangle=0$ for $w \in U M_{p}$ with $\left\langle X_{1}, w\right\rangle=0$. So $X_{1}$ is an eigenvector of $A_{\varphi X_{1}}$. Hence, since $A_{\varphi X_{1}}$ is symmetric we can also choose $X_{2}$ and $X_{3}$ as eigenvectors of $A_{\varphi X_{1}}$. If $X_{2}$ and $X_{3}$ are both eigenvectors of $A_{\varphi X_{1}}$ with the same eigenvalue, we choose $X_{2}$ as the vector in which the function $f$ restricted to $\left\{u \in U M_{p}:\left\langle u, X_{1}\right\rangle=0\right\}$ attains an absolute maximum. So, in this case, we find that $\left\langle h\left(X_{2}, X_{2}\right), \varphi X_{3}\right\rangle=0$. Furthermore, we may still assume that $X_{2}$ and $X_{3}$ satisfy the following two properties, see [2]. $\left\langle h\left(X_{2}, X_{2}\right), \varphi X_{2}\right\rangle \geq 0, \quad\left\langle h\left(X_{2}, X_{2}\right), \varphi X_{2}\right\rangle^{2} \geq\left\langle h\left(X_{3}, X_{3}\right), \varphi X_{3}\right\rangle^{2}$. Then with respect to such an orthonormal basis $\left\{X_{1}, X_{2}, X_{3}\right\}$, we can write the Weingarten maps $A_{i}=A_{\xi_{i}}\left(\xi_{i}=\varphi X_{i} i=1,2,3\right)$ at the point $p \in M$ in the following way:

$$
A_{1}=\left[\begin{array}{ccc}
\lambda_{1} & 0 & 0 \\
0 & \lambda_{2} & 0 \\
0 & 0 & \lambda_{3}
\end{array}\right], A_{2}=\left[\begin{array}{ccc}
0 & \lambda_{2} & 0 \\
\lambda_{2} & a & b \\
0 & b & c
\end{array}\right], A_{3}=\left[\begin{array}{ccc}
0 & 0 & \lambda_{3} \\
0 & b & c \\
\lambda_{3} & c & d
\end{array}\right]
$$

If the eigenvalues of $A_{1}$ have constant multiplicity on a neighborhood of $p$ we extend this basis differentiably about $p$ and define the open dense 
set $U$ by this property. Note that we have, from the above arguments,

$$
\lambda_{1}, a \geq 0, a^{2} \geq d^{2} \text { at } p .
$$

Also at the point $p$, since $f$ attains an absolute maximum in the direction $X_{1}$, we know that the function $f_{2}$ defined by $f_{2}(\theta)=f\left(\cos \theta X_{1}+\right.$ $\left.\sin \theta X_{i}\right), i=2,3$ has a relative maximum at the origin. Hence $f_{2}^{\prime \prime}(0) \leq 0$, which implies that

$$
\lambda_{1} \geq 2 \lambda_{2}, \lambda_{1} \geq 2 \lambda_{3} \quad \text { at } \quad p
$$

We remark that if $\lambda_{2}=\lambda_{3}$ on a component of $U$ we can choose the basis in such a way that $b=0$ on this component.

We now consider the hypothesis that $M$ is 2-type and mass-symmetric. Let $\Delta$ be the Laplacian of $M$ associated with the induced metric. This Laplacian can be extended componentwise to $E^{8}$-valued smooth maps $u$ of $M$ as follows:

$$
\Delta u=\sum_{i=1}^{3}\left(\bar{\nabla}_{\nabla_{X_{i}} X_{i}} u-\bar{\nabla}_{X_{i}} \bar{\nabla}_{X_{i}} u\right)
$$

The position vector $x$ of $M$ with respect to the origin of $E^{8}$ is given by the immersion (2.3) and can be written as follows:

$$
x=x_{1}+x_{2}, \Delta x_{1}=\mu_{1} x_{1}, \Delta x_{2}=\mu_{2} x_{2}
$$

where $x_{1}, x_{2}$ are non-constant $E^{8}$-valued maps on $M$. Note that $A_{x}=-I$ and $D x=0$. Moreover, since $H=\sum_{i=1}^{3} \frac{1}{3}\left(\operatorname{tr} A_{i}\right) \xi_{i}-x$ and $\Delta x=-3 H$, by using (3.5) we find

$$
\Delta H=\left(\mu_{1}+\mu_{2}\right) \sum_{i=1}^{3} \alpha_{i} \xi_{i}+\left(\frac{\mu_{1} \mu_{2}}{3}-\left(\mu_{1}+\mu_{2}\right)\right) x
$$

where $\alpha_{i}=\frac{1}{3} \operatorname{tr} A_{i}, i=1,2,3$. On the other hand applying (3.4) to $H$ we have, by direct computation

$$
\begin{gathered}
\Delta H=\sum_{i, j}\left[\left(\Delta \alpha_{i}\right) \xi_{i}+\alpha_{i} \Delta^{D} \xi_{i}+2 A_{i} \operatorname{grad} \alpha_{i}+\alpha_{i}\left(\nabla_{X_{j}} A_{i}\right) X_{j}\right. \\
\quad-\alpha_{i}\left(\operatorname{tr} A_{i} A_{j}\right) \xi_{j}-2 D_{\operatorname{grad} \alpha_{i}} \xi_{i} \\
\left.+\alpha_{i} A_{D_{X_{j}} \xi_{i}} X_{j}+3 \alpha_{i} \xi_{i}-3\|H\|^{2} x\right]
\end{gathered}
$$


where

$$
\Delta^{D} \xi_{i}=\sum_{j=1}^{3}\left(D_{\nabla_{X_{j}} X_{j}} \xi_{i}-D_{X_{j}} D_{X_{j}} \xi_{i}\right)
$$

Since $D x=0$, we have that $D \xi_{i}$ is perpendicular to $x$. So $\left\langle\Delta^{D} \xi_{i}, x\right\rangle=0$. Now combining (3.6) with (3.7) we obtain from the $x$-component that $\|H\|^{2}=\frac{1}{3}\left(\mu_{1}+\mu_{2}\right)-\frac{\mu_{1} \mu_{2}}{9}$. Thus $M$ has constant mean curvature, (for this well-known result, see $[6$, p. 274]) and so we have

$$
\alpha_{1}^{2}+\alpha_{2}^{2}+\alpha_{3}^{2}=\text { const. }
$$

Also, from the tangential component we have

$$
\sum_{i, j=1}^{3}\left(2 A_{j} \operatorname{grad} \alpha_{j}+\alpha_{j}\left(\nabla_{X_{i}} A_{j}\right) X_{i}+\alpha_{j} A_{D_{X_{i}} \xi_{j}} X_{i}\right)=0 .
$$

Now using the Codazzi equation

$$
\left(\nabla_{X_{i}} A_{s}\right) X_{j}-A_{D_{X_{i}} \xi_{s}} X_{j}-\left(\nabla_{X_{j}} A_{s}\right) X_{i}+A_{D_{X_{j}} \xi_{s}} X_{i}=0
$$

we obtain

$$
\operatorname{grad} \operatorname{tr} A_{s}-\sum_{i, j=1}^{3}\left(\operatorname{tr} A_{i} w_{s}^{i}\left(X_{j}\right) X_{j}+\left(\nabla_{X_{j}} A_{s}\right) X_{j}-A_{D_{X_{j}} \xi_{s}} X_{j}\right)=0 .
$$

From (3.9),

$$
\sum_{i=1}^{3}\left(\operatorname{tr} A_{i}\right) \operatorname{grad} \operatorname{tr} A_{i}=0 .
$$

Using (3.12) we obtain

$$
\sum_{i, j} \alpha_{j}\left(\nabla_{X_{i}} A_{j}\right) X_{i}=\sum_{i, j} \alpha_{j} A_{D_{X_{i}} \xi_{j}} X_{i}
$$

Thus (3.10) becomes $\sum_{i, j}\left(A_{i} \operatorname{grad} \alpha_{i}+\alpha_{i} A_{D_{X_{j}} \xi_{i}} X_{j}\right)=0$, or

$$
\sum_{i, j} A_{i}\left(\beta_{i}^{j} X_{j}\right)=0
$$


where for convenience we have put

$$
\beta_{j}^{i}=X_{i} \alpha_{j}-\sum_{k} \alpha_{k} w_{j}^{k}\left(X_{i}\right)
$$

Now from (3.1) and (3.14) we obtain the following useful equations

$$
\begin{aligned}
& \lambda_{1} \beta_{1}^{1}+\lambda_{2} \beta_{2}^{2}+\lambda_{3} \beta_{3}^{3}=0 \\
& \lambda_{2} \beta_{1}^{2}+\lambda_{2} \beta_{2}^{1}+a \beta_{2}^{2}+b \beta_{2}^{3}+b \beta_{3}^{2}+c \beta_{3}^{3}=0, \\
& \lambda_{3} \beta_{1}^{3}+b \beta_{2}^{2}+c \beta_{2}^{3}+\lambda_{3} \beta_{3}^{1}+c \beta_{3}^{2}+d \beta_{3}^{3}=0 .
\end{aligned}
$$

We continue with some further calculations. Combining the $\xi$-component of (3.6) and (3.7) we obtain

$$
\sum_{i=1}^{3}\left\langle\alpha_{i} \Delta^{D} \xi_{i}-2 D_{\operatorname{grad} \alpha_{i}} \xi_{i}, \xi\right\rangle=0
$$

which by direct computation becomes

$$
\sum_{i=1}^{3} \beta_{i}^{i}=0 .
$$

Also (3.13) gives

$$
\sum_{i=1}^{3} \alpha_{i} \beta_{i}^{j}=0, j=1,2,3 .
$$

Finally, combining the $\xi_{i}$-components, $i=1,2,3$, of (3.6) and (3.7) we have the following.

From the $\xi_{1}$-component

$$
\begin{aligned}
\Delta \alpha_{1}+ & \sum_{i, j}\left[\alpha_{1}\left(1+w_{1}^{2}\left(X_{i}\right)^{2}+w_{1}^{3}\left(X_{i}\right)^{2}\right)\right. \\
& +\alpha_{2}\left(X_{i} w_{1}^{2}\left(X_{i}\right)-w_{1}^{2}\left(X_{j}\right) w_{i}^{j}\left(X_{i}\right)+w_{1}^{3}\left(X_{i}\right) w_{2}^{3}\left(X_{i}\right)\right) \\
& +\alpha_{3}\left(X_{i} w_{1}^{3}\left(X_{i}\right)-w_{1}^{3}\left(X_{j}\right) w_{i}^{j}\left(X_{i}\right)-w_{1}^{2}\left(X_{i}\right) w_{2}^{3}\left(X_{i}\right)\right) \\
& \left.+2\left(X_{i} \alpha_{j}\right) w_{1}^{j}\left(X_{i}\right)-\alpha_{i} \operatorname{tr} A_{i} A_{1}\right]=\alpha_{1}\left(\mu_{1}+\mu_{2}-3\right) .
\end{aligned}
$$

From the $\xi_{2}$-component

$$
\begin{aligned}
\Delta \alpha_{2}+ & \sum_{i, j}\left[\alpha_{1}\left(w_{1}^{2}\left(X_{j}\right) w_{i}^{j}\left(X_{i}\right)-X_{i} w_{1}^{2}\left(X_{i}\right)+w_{1}^{3}\left(X_{i}\right) w_{2}^{3}\left(X_{i}\right)\right)\right. \\
& +\alpha_{2}\left(1+w_{1}^{2}\left(X_{i}\right)^{2}+w_{2}^{3}\left(X_{i}\right)^{2}\right)
\end{aligned}
$$




$$
\begin{aligned}
& +\alpha_{3}\left(X_{i} w_{2}^{3}\left(X_{i}\right)-w_{2}^{3}\left(X_{j}\right) w_{i}^{j}\left(X_{i}\right)+w_{1}^{2}\left(X_{i}\right) w_{1}^{3}\left(X_{i}\right)\right) \\
& \left.+2\left(X_{i} \alpha_{j}\right) w_{2}^{j}\left(X_{i}\right)-\alpha_{i} \operatorname{tr} A_{i} A_{2}\right]=\alpha_{1}\left(\mu_{1}+\mu_{2}-3\right) .
\end{aligned}
$$

From the $\xi_{3}$-component

$$
\begin{aligned}
\Delta \alpha_{3}+ & \sum_{i, j}\left[\alpha_{1}\left(X_{i} w_{3}^{1}\left(X_{i}\right)+w_{1}^{3}\left(X_{j}\right) w_{i}^{j}\left(X_{i}\right)-w_{1}^{2}\left(X_{i}\right) w_{2}^{3}\left(X_{i}\right)\right)\right. \\
& +\alpha_{2}\left(X_{i} w_{3}^{2}\left(X_{i}\right)+w_{1}^{2}\left(X_{i}\right) w_{1}^{3}\left(X_{i}\right)+w_{2}^{3}\left(X_{j}\right) w_{i}^{j}\left(X_{i}\right)\right) \\
& +\alpha_{3}\left(1+w_{1}^{3}\left(X_{i}\right)^{2}+w_{2}^{3}\left(X_{i}\right)^{2}\right) \\
& \left.+2\left(X_{i} \alpha_{j}\right) w_{3}^{j}\left(X_{i}\right)-\alpha_{i} \operatorname{tr} A_{i} A_{3}\right]=\alpha_{3}\left(\mu_{1}+\mu_{2}-3\right)
\end{aligned}
$$

Now set

$$
\begin{aligned}
& E_{1}\left(X_{i}\right)=X_{i} \lambda_{1} \\
& E_{2}\left(X_{i}\right)=\left(\lambda_{1}-2 \lambda_{2}\right) w_{1}^{2}\left(X_{i}\right) \\
& E_{3}\left(X_{i}\right)=\left(\lambda_{1}-2 \lambda_{3}\right) w_{1}^{3}\left(X_{i}\right) \\
& E_{4}\left(X_{i}\right)=\left(\lambda_{2}-\lambda_{3}\right) w_{2}^{3}\left(X_{i}\right)-b w_{1}^{2}\left(X_{i}\right)-c w_{1}^{3}\left(X_{i}\right) \\
& E_{5}\left(X_{i}\right)=X_{i} \lambda_{2}-a w_{1}^{2}\left(X_{i}\right)-b w_{1}^{3}\left(X_{i}\right) \\
& E_{6}\left(X_{i}\right)=X_{i} \lambda_{3}-c w_{1}^{2}\left(X_{i}\right)-d w_{1}^{3}\left(X_{i}\right) \\
& E_{7}\left(X_{i}\right)=X_{i} a+3 \lambda_{2} w_{1}^{2}\left(X_{i}\right)-3 b w_{2}^{3}\left(X_{i}\right) \\
& E_{8}\left(X_{i}\right)=X_{i} b+\lambda_{2} w_{1}^{3}\left(X_{i}\right)+(a-2 c) w_{2}^{3}\left(X_{i}\right) \\
& E_{9}\left(X_{i}\right)=X_{i} c+\lambda_{3} w_{1}^{2}\left(X_{i}\right)+(2 b-d) w_{2}^{3}\left(X_{i}\right) \\
& E_{10}\left(X_{i}\right)=X_{i} d+3 \lambda_{3} w_{1}^{3}\left(X_{i}\right)+3 c w_{2}^{3}\left(X_{i}\right) .
\end{aligned}
$$

Applying the Codazzi equation (3.11) successively for all values of $(s, i, j)$ we obtain

(i) $\quad E_{1}\left(X_{2}\right)=E_{2}\left(X_{1}\right)$

(ii) $\quad E_{1}\left(X_{3}\right)=E_{3}\left(X_{1}\right)$

(iii) $\quad E_{2}\left(X_{2}\right)=E_{5}\left(X_{1}\right)$

(iv) $E_{2}\left(X_{3}\right)=E_{4}\left(X_{1}\right)$

(v) $\quad E_{3}\left(X_{2}\right)=E_{4}\left(X_{1}\right)$

(vi) $E_{3}\left(X_{3}\right)=E_{6}\left(X_{1}\right)$

(vii) $E_{4}\left(X_{2}\right)=E_{5}\left(X_{3}\right)$

(viii) $E_{4}\left(X_{3}\right)=E_{6}\left(X_{2}\right)$

(ix) $E_{5}\left(X_{2}\right)=E_{7}\left(X_{1}\right)$

(x) $\quad E_{5}\left(X_{3}\right)=E_{8}\left(X_{1}\right)$

(xi) $\quad E_{6}\left(X_{2}\right)=E_{9}\left(X_{1}\right)$

(xii) $\quad E_{6}\left(X_{3}\right)=E_{10}\left(X_{1}\right)$

(xiii) $E_{7}\left(X_{3}\right)=E_{8}\left(X_{2}\right)$

(xiv) $E_{8}\left(X_{3}\right)=E_{9}\left(X_{2}\right)$

(xv) $\quad E_{8}\left(X_{3}\right)=E_{10}\left(X_{2}\right)$ 
and in addition

$$
\begin{aligned}
& 3 \beta_{1}^{i}=E_{1}\left(X_{i}\right)+E_{5}\left(X_{i}\right)+E_{6}\left(X_{i}\right) \\
& 3 \beta_{2}^{i}=E_{2}\left(X_{i}\right)+E_{7}\left(X_{i}\right)+E_{9}\left(X_{i}\right) \\
& 3 \beta_{3}^{i}=E_{3}\left(X_{i}\right)+E_{8}\left(X_{i}\right)+E_{10}\left(X_{i}\right) .
\end{aligned}
$$

Thus by using (3.23) we observe that $\beta_{i}^{j}=\beta_{j}^{i}, i, j=1,2,3$.

\section{Proof of the Theorem}

Now we assume that $M$ is flat and continue with some more calculations. From (2.6) and (3.1) we obtain

$$
\begin{aligned}
& 1+\lambda_{1} \lambda_{2}-\lambda_{2}^{2}=0 \\
& 1+\lambda_{1} \lambda_{3}-\lambda_{3}^{2}=0 \\
& 1+\lambda_{2} \lambda_{3}+a c+b d-b^{2}-c^{2}=0
\end{aligned}
$$

and from these $\left(\lambda_{2}-\lambda_{3}\right)\left(\lambda_{1}-\lambda_{2}-\lambda_{3}\right)=0$. If $\lambda_{2} \neq \lambda_{3}$, then $\lambda_{1}=\lambda_{2}+\lambda_{3}$. But from (3.3) we obtain $\lambda_{2}+\lambda_{3} \geq 2 \lambda_{2}$ and $\lambda_{2}+\lambda_{3} \geq 2 \lambda_{3}$ at $p$. Thus $\lambda_{2}=\lambda_{3}$, a contradiction. So $\lambda_{2}=\lambda_{3}=\lambda$ and then, from our initial discussion in Section 3, we have also $b=0$. Now (4.1) becomes

$$
\begin{aligned}
& 1+\lambda_{1} \lambda-\lambda^{2}=0 \\
& 1+\lambda^{2}+a c-c^{2}=0 .
\end{aligned}
$$

From these relations we have that $\lambda \neq 0, \lambda_{1} \neq \lambda, \lambda_{1} \neq 2 \lambda, c \neq 0$ and $a \neq c$. Also using (3.3) we have $-1 \leq \lambda<0$ at $p$.

Now from (3.16) and (3.17) we obtain $\beta_{1}^{1}=0$ and $\beta_{3}^{3}=-\beta_{2}^{2}$.

Also, since $\Sigma_{i} \alpha_{i}^{2} \neq 0$ and $\beta_{i}^{j}=\beta_{j}^{i}$, from (3.18) we get

$$
\beta_{2}^{2}\left[\left(\beta_{1}^{2}\right)^{2}-\left(\beta_{1}^{3}\right)^{2}+\frac{c-a}{\lambda} \beta_{1}^{3} \beta_{2}^{3}\right]=0 .
$$

Lemma $1 \beta_{2}^{2}=-\beta_{3}^{3}=0$.

Proof. Suppose $\beta_{2}^{2} \neq 0$. Then from (4.3) we have

$$
\left(\beta_{1}^{2}\right)^{2}-\left(\beta_{1}^{3}\right)^{2}+\frac{c-a}{\lambda} \beta_{1}^{3} \beta_{2}^{3}=0 .
$$


From (3.18) by using (3.16) we obtain

$$
\begin{aligned}
& \left(c^{2}-a^{2}+d^{2}\right) \beta_{2}^{2}-2 c d \beta_{2}^{3}=0, \\
& \left(\left(3 \lambda^{2}-1\right)(c-a)+2 \lambda^{2}(a+c)\right) \beta_{2}^{2}+2 \lambda^{2} d \beta_{2}^{3}=0, \\
& \left(\lambda^{2}-1\right) d \beta_{2}^{2}+\left(2 \lambda^{2}(a+c)-2 c\left(3 \lambda^{2}-1\right)\right) \beta_{2}^{3}=0 .
\end{aligned}
$$

If $d=0$, from (4.5) we have $a+c=0$ and $3 \lambda^{2}-1=0$. Thus $\alpha_{1}=\alpha_{2}=$ $\alpha_{3}=0$, a contradiction.

Thus $d \neq 0$, and from (4.5) we have $\beta_{2}^{3}=\frac{c^{2}-a^{2}+d^{2}}{2 c d} \beta_{2}^{2}$, while using (4.4) and (3.16) we obtain

$$
d^{2}=\frac{(a+c)^{2}(a-2 c)}{a} .
$$

Also from (4.5) we obtain $\lambda^{2}=\frac{a}{a-2 c}$ and from (4.1) $a=\frac{2\left(c^{2}-1\right)}{c}$. Thus finally we have

$$
\lambda^{2}=1-c^{2}, \quad a=\frac{2\left(c^{2}-1\right)}{c}, \quad d^{2}=\frac{\left(3 c^{2}-2\right)^{2}}{c^{2}\left(1-c^{2}\right)}
$$

and from (3.9) we conclude that $\lambda, a, c, d$ are constant.

Now from (3.15) we obtain

$$
\begin{aligned}
& 3 \beta_{1}^{i}=-\frac{3 c^{2}-2}{c} w_{1}^{2}\left(X_{i}\right)-d w_{1}^{3}\left(X_{i}\right), \\
& 3 \beta_{2}^{i}=-\frac{3 c^{2}-2}{\lambda} w_{1}^{2}\left(X_{i}\right)-d w_{2}^{3}\left(X_{i}\right), \\
& 3 \beta_{3}^{i}=-\frac{3 c^{2}-2}{\lambda} w_{1}^{3}\left(X_{i}\right)+\frac{3 c^{2}-2}{c} w_{2}^{3}\left(X_{i}\right) .
\end{aligned}
$$

From (4.6) and (3.16) we obtain

$$
\beta_{1}^{2}=\frac{2-c^{2}}{2 \lambda c} \beta_{2}^{2}, \quad \beta_{2}^{3}=\frac{c\left(3 c^{2}-2\right)}{2 d\left(1-c^{2}\right)} \beta_{2}^{2}, \quad \beta_{1}^{3}=\frac{\left(c^{2}-2\right)\left(3 c^{2}-2\right)}{2 \lambda c^{2} d} \beta_{2}^{2} .
$$

Now from $\left(3.23\right.$, (i), (ii)) we get $w_{1}^{2}\left(X_{1}\right)=0, w_{1}^{3}\left(X_{1}\right)=0$ and then from $\left(3.23\right.$, (iii), (vi)), $w_{1}^{2}\left(X_{2}\right)=w_{1}^{3}\left(X_{3}\right)=0$. Thus we have $\beta_{1}^{2}=$ $-d w_{1}^{3}\left(X_{2}\right), \beta_{2}^{2}=-d w_{2}^{3}\left(X_{2}\right)$ and $\beta_{2}^{3}=-\frac{3 c^{2}-2}{\lambda} w_{1}^{2}\left(X_{3}\right)-d w_{2}^{3}\left(X_{3}\right)$. Therefore

$$
w_{1}^{3}\left(X_{2}\right)=\frac{2-c^{2}}{2 \lambda c} w_{2}^{3}\left(X_{2}\right)
$$


and

$$
\left(3 c^{2}-2\right) w_{1}^{2}\left(X_{3}\right)+\lambda d w_{2}^{3}\left(X_{3}\right)=\frac{\lambda c\left(3 c^{2}-2\right)}{2\left(1-c^{2}\right)} w_{2}^{3}\left(X_{2}\right)
$$

Also $3 \beta_{1}^{3}=-\frac{3 c^{2}-2}{c} w_{1}^{2}\left(X_{3}\right)$ and $3 \beta_{3}^{1}=\frac{3 c^{2}-2}{c} w_{2}^{3}\left(X_{1}\right)$. Thus $w_{2}^{3}\left(X_{1}\right)=$ $-w_{1}^{2}\left(X_{3}\right)$ and from $\beta_{1}^{2}=\beta_{2}^{1}$ we get $w_{2}^{3}\left(X_{1}\right)=w_{1}^{3}\left(X_{2}\right)$. Then

$$
w_{1}^{2}\left(X_{3}\right)=\frac{c^{2}-2}{2 \lambda c} w_{2}^{3}\left(X_{2}\right)
$$

From $(3.22)$ we obtain $E_{8}\left(X_{2}\right)=-\frac{c^{2}+2}{2 c} w_{2}^{3}\left(X_{2}\right)$ and $E_{10}\left(X_{2}\right)=3 \frac{c^{2}+2}{2 c} w_{2}^{3}\left(X_{2}\right)$. Thus $E_{10}\left(X_{2}\right)=-3 E_{8}\left(X_{2}\right)$. Now, using $\left(3.23\right.$, (xiii), (xv)) we have $E_{9}\left(X_{3}\right)$ $=-3 E_{7}\left(X_{3}\right)$ or

$$
10 \lambda w_{1}^{2}\left(X_{3}\right)-d w_{2}^{3}\left(X_{3}\right)=0 .
$$

Finally from (4.6), (4.7) and (4.8) we have $\left(5 c^{4}-12 c^{2}+8\right) w_{2}^{3}\left(X_{2}\right)=0$. But $5 c^{4}-12 c^{2}+8 \neq 0$. Thus $w_{2}^{3}\left(X_{2}\right)=0$ and so $\beta_{2}^{2}=0$, a contradiction.

Lemma $2 \lambda, a, c, d$ are constant and $w_{i}^{j}=0, i, j=1,2,3$ on $M$.

Proof. We have $\beta_{1}^{1}=\beta_{2}^{2}=\beta_{3}^{3}=0$ and from (3.16) and (3.18) we get $\beta_{1}^{2}=0, d \beta_{1}^{3}=0, d \beta_{2}^{3}=0$ and

$$
\lambda \beta_{1}^{3}+c \beta_{2}^{3}=0, \quad\left(3 \lambda^{2}-1\right) \beta_{1}^{3}+\lambda(a+c) \beta_{2}^{3}=0 .
$$

We will examine two cases, $d \neq 0$ and $d=0$.

Case I: $\quad d \neq 0$

We have $\beta_{1}^{3}=\beta_{2}^{3}=0$. Thus $\beta_{i}^{j}=0, i, j,=1,2,3$. From (3.15) we get

$$
\frac{3 \lambda^{2}+1}{\lambda^{2}} X_{i} \lambda=(a+c) w_{1}^{2}\left(X_{i}\right)+d w_{1}^{3}\left(X_{i}\right)
$$

and from $(3.23$, (iv), (v))

$$
\begin{aligned}
& \left(\lambda^{2}+1\right) w_{1}^{2}\left(X_{3}\right)=\lambda c w_{1}^{3}\left(X_{1}\right) \\
& w_{1}^{2}\left(X_{3}\right)=w_{1}^{3}\left(X_{2}\right) .
\end{aligned}
$$

Now using (4.2), (4.11) and (4.12), from (3.23, (vii)) we obtain

$$
\left(1-\lambda^{2}\right)\left(\lambda^{2}-2 c^{2}+1\right) w_{1}^{2}\left(X_{3}\right)=0
$$


We claim that $\left(1-\lambda^{2}\right)\left(\lambda^{2}-2 c^{2}+1\right) \neq 0$.

Indeed, let $\lambda^{2}=1$, or $\lambda=-1$ since $\lambda$ is negative. Then from (4.2) we have $a=\frac{c^{2}-2}{c}$ and from (3.22) $E_{1}=0$. Now from $(3.23$, (i), (ii)) we get $w_{1}^{2}\left(X_{1}\right)=w_{1}^{3}\left(X_{1}\right)=0$ and then from (4.12) and $\left(3.23\right.$, (iii) $w_{1}^{2}\left(X_{3}\right)=$ $w_{1}^{3}\left(X_{2}\right)=w_{1}^{2}\left(X_{2}\right)=0$. After these from (4.11) we also have $w_{1}^{3}\left(X_{3}\right)=0$. Thus $w_{1}^{2}=w_{1}^{3}=0$.

Now from (3.19) we obtain $\left(3 \lambda^{2}-1\right)\left(4-\lambda_{1}^{2}-2 \lambda^{2}-\mu_{1}-\mu_{2}\right)-\lambda^{2}(a+c)^{2}-$ $\lambda^{2} d^{2}=0$. This relation with (3.9) and (4.2) imply that $a, c, d$ are constant. Now from $(3.23,(\mathrm{x}),(\mathrm{xiii}),(\mathrm{xiv}))$ we get $w_{2}^{3}=0$. Thus $w_{i}^{j}=0, i, j=1,2,3$ and then (3.19), (3.20), (3.21) become

$$
\begin{aligned}
& 2+a c+c^{2}+d^{2}+2 z=0, \quad(a+c)\left(3+a^{2}+c^{2}+z\right)+c d^{2}=0, \\
& 3+a c+3 c^{2}+d^{2}+z=0
\end{aligned}
$$

where $z=\mu_{1}+\mu_{2}-3$.

We can easily see that this system together with (4.2) is impossible. Thus $\lambda^{2} \neq 1$.

Suppose now that $\lambda^{2}-2 c^{2}+1=0$. Then from (4.2) we get $a+c=0$. Thus from (3.15) we have

$$
\begin{aligned}
& X_{i} \lambda=\frac{\lambda^{2} d}{3 \lambda^{2}+1} w_{1}^{3}\left(X_{i}\right),\left(3 \lambda^{2}-1\right) w_{1}^{2}=\lambda d w_{2}^{3}, \\
& X_{i} d=-\frac{3 \lambda^{2}-1}{\lambda} w_{1}^{3}\left(X_{i}\right) .
\end{aligned}
$$

Now from (3.23, (i), (ii), (iv), (v)) we get

$$
\begin{aligned}
& X_{2} \lambda=-\lambda w_{1}^{2}\left(X_{1}\right), \quad X_{3} \lambda=-\lambda w_{1}^{3}\left(X_{1}\right) \\
& \left(\lambda^{2}+1\right) w_{1}^{2}\left(X_{3}\right)=\lambda c w_{1}^{3}\left(X_{1}\right), \quad w_{1}^{2}\left(X_{3}\right)=w_{1}^{3}\left(X_{2}\right)
\end{aligned}
$$

By using (4.14) and (4.15), from $(3.23,(\mathrm{x}))$ we obtain $\left(3 \lambda^{2}-1\right) w_{1}^{2}\left(X_{1}\right)-$ $\lambda d w_{1}^{2}\left(X_{3}\right)=0$, while from $(3.23$, (ix), (xi)) we conclude that $\left(3 \lambda^{2}+1\right) w_{1}^{2}\left(X_{1}\right)+\lambda d w_{1}^{2}\left(X_{3}\right)=0$. From these two relations we have $w_{1}^{2}\left(X_{1}\right)=w_{1}^{2}\left(X_{3}\right)=0$. Now from (4.15) we have $w_{1}^{3}\left(X_{1}\right)=w_{1}^{3}\left(X_{2}\right)=0$ and from $\left(3.23\right.$, (iii) $w_{1}^{2}\left(X_{2}\right)=0$. Thus $w_{1}^{2}=0$ and now from (4.14) we get $w_{2}^{3}=0$. Therefore $(3.20)$ becomes $c d^{2}=0$, a contradiction.

Thus finally we have $\left(1-\lambda^{2}\right)\left(\lambda^{2}-2 c^{2}+1\right) \neq 0$ and from (4.13) we have $w_{1}^{2}\left(X_{3}\right)=0$, while from (4.12) $w_{1}^{3}\left(X_{1}\right)=w_{1}^{3}\left(X_{2}\right)=0$. Also from (4.11) and (3.23), (ii), (vi), (viii)) we find $w_{1}^{3}\left(X_{3}\right)=0, w_{1}^{2}\left(X_{1}\right)=0$ and $w_{1}^{2}\left(X_{2}\right)=0$. 
Thus we have $w_{1}^{2}=w_{1}^{3}=0$. Now from (4.11), $\lambda=$ const. and from (3.19)

$$
\left(\lambda_{1}+2 \lambda\right)\left(4-\lambda_{1}^{2}-2 \lambda^{2}-\mu_{1}-\mu_{2}\right)-\lambda(a+c)^{2}-\lambda d^{2}=0 .
$$

From this, (3.9) and (4.2) we conclude that $a, c, d$ are constant.

Finally from (3.15) we obtain $w_{2}^{3}=0$.

Case II: $\quad d=0$

The determinant of (4.10) is $D=\frac{1}{c}\left(\lambda^{4}+\left(1+c^{2}\right) \lambda^{2}-c^{2}\right)$.

If $D=0$, using (3.9) and (4.2) we conclude that $\lambda, a, c$ are constant. Thus from (3.15) we have

$$
\beta_{1}^{i}=\alpha_{2} w_{2}^{1}\left(X_{i}\right), \quad \beta_{2}^{i}=\alpha_{1} w_{1}^{2}\left(X_{i}\right), \quad \beta_{3}^{i}=\alpha_{1} w_{1}^{3}\left(X_{i}\right)+\alpha_{2} w_{2}^{3}\left(X_{i}\right)
$$

and since $\alpha_{1}^{2}+\alpha_{2}^{2} \neq 0$ we obtain $w_{1}^{2}\left(X_{1}\right)=w_{1}^{2}\left(X_{2}\right)=0$. Now from (3.23, (ii), (iv)), $w_{1}^{3}\left(X_{1}\right)=0$ and then $w_{1}^{2}\left(X_{3}\right)=0$. So $w_{1}^{2}=0$. From $(3.23$, (vii), (viii)) we obtain $w_{1}^{3}\left(X_{2}\right)=w_{1}^{3}\left(X_{3}\right)=0$. Thus $w_{1}^{3}=0$. Using $w_{1}^{2}=w_{1}^{3}=0$, from $\left(3.23\right.$, (ix), (xiii), (xiv)) we conclude that $w_{2}^{3}=0$.

Now suppose $D \neq 0$. In this case from (4.10) we have $\beta_{i}^{j}=0$ for $i, j=1,2,3$. Then from (3.15),

$$
X_{i} \alpha_{1}=\alpha_{2} w_{1}^{2}\left(X_{i}\right), X_{i} \alpha_{2}=\alpha_{1} w_{2}^{1}\left(X_{i}\right), \alpha_{1} w_{1}^{3}+\alpha_{2} w_{2}^{3}=0 .
$$

We assert that $\alpha_{1} \neq 0$. Indeed, if for the moment we suppose that $\alpha_{1}=0$, then from (4.16) we have $w_{1}^{2}=w_{2}^{3}=0$. Now from (3.23, (iv), (vii), (viii)) we obtain $w_{1}^{3}=0$ and from (3.19) we get $\lambda(a+c)^{2}=0$, a contradiction.

For $\alpha_{2}$ we distinguish two subcases $\mathrm{II}_{1}, \alpha_{2}=0$ and $\mathrm{II}_{2}, \alpha_{2} \neq 0$, which we examine separately.

II $_{1}: \alpha_{2}=0$.

In this case we have $a+c=0$ and $\lambda=$ const. So from (4.1) we have that $a, c$ are constant, while from (4.16) $w_{1}^{2}=w_{1}^{3}=0$. Now from $(3.23,(\mathrm{x})$, (xiii), (xiv)) we get $w_{2}^{3}=0$.

$\mathbf{I I}_{2}: \quad \alpha_{2} \neq 0$.

Now we have $\alpha_{1} \alpha_{2} \neq 0$. From (4.16) and (3.23, (i), (iii)) we obtain

$$
\begin{aligned}
& \left(3 \lambda^{2}+1\right) w_{1}^{2}\left(X_{1}\right)+\lambda(a+c) w_{1}^{2}\left(X_{2}\right)=0, \\
& \lambda\left(\left(2 \lambda^{2}+1\right) a-\lambda^{2} c\right) w_{1}^{2}\left(X_{1}\right)-\left(3 \lambda^{2}+1\right)\left(\lambda^{2}+1\right) w_{1}^{2}\left(X_{2}\right)=0 .
\end{aligned}
$$

The determinant of this system is

$$
D=\left(3 \lambda^{2}+1\right)^{2}\left(\lambda^{2}+1\right)+\lambda^{2}(a+c)\left(\left(2 \lambda^{2}+1\right) a-\lambda^{2} c\right) .
$$


If $D=0$, then using (3.9) and (4.2) we have that $\lambda, a, c$ are constant. Now from (4.16) we have $w_{1}^{2}=0$ and from $(3.23$, (iii), (v), (vi)) we obtain $w_{1}^{3}=0$. Finally from $(4.16) w_{2}^{3}=0$. Now suppose $D \neq 0$. Then $w_{1}^{3}\left(X_{1}\right)=$ $w_{1}^{2}\left(X_{2}\right)=0$ and $X_{1} \lambda=X_{2} \lambda=0$. Now from $(3.23,($ vi $))$ we have $w_{1}^{3}\left(X_{3}\right)=0$. From (4.16) and $(3.23,($ ii), (iv)) we obtain

$$
\begin{aligned}
& \left(3 \lambda^{2}+1\right) w_{1}^{3}\left(X_{1}\right)+\lambda(a+c) w_{1}^{2}\left(X_{3}\right)=0, \\
& \lambda c w_{1}^{3}\left(X_{1}\right)-\left(\lambda^{2}+1\right) w_{1}^{2}\left(X_{3}\right)=0 .
\end{aligned}
$$

If for the determinant $D_{1}=\left(\lambda^{2}+1\right)\left(3 \lambda^{2}+1\right)+\lambda^{2} c(a+c)$, we have $D_{1}=0$, then from (3.9) and (4.2) we obtain $\lambda, a, c=$ constant. So, from (4.16) and $(3.23,(\mathrm{v}))$ we conclude that $w_{1}^{3}=w_{2}^{3}=0$. If $D_{1} \neq 0$, then $w_{1}^{3}\left(X_{1}\right)=$ $w_{1}^{2}\left(X_{3}\right)=0$. Thus $w_{1}^{2}=0$ and from (4.16) $\lambda=$ constant and $a+c=$ const. Now from (4.2) we have $a, c$ are constant. Again from $(3.23,(\mathrm{v}))$ we have $w_{1}^{3}\left(X_{2}\right)=0$. So $w_{1}^{3}=0$ and from (4.16) $w_{2}^{3}=0$, which completes the proof of the lemma.

Proof of the Theorem. From Lemma 2 we have that $\lambda, a, c, d$ are constant and $w_{i}^{j}=0, i, j=1,2,3$. Thus from (3.22) we have $E_{1}=E_{2}=\ldots=E_{10}=$ 0 . Now, according to [2], we have that $M$ is $C$-parallel and the theorem follows from Proposition 5.2 of [2] which says that under these conditions the position vector is as in the statement of the Theorem and $M$ lies fully in $E^{8}$.

We close this paper with an auxiliary result for which we need the following definition (see $[8$, p. 20]).

Definition If $\gamma(s)$ is a curve in a Reimannian manifold $N$, parametrized by arc length $s$, we say that $\gamma$ is a Frenet curve of order $r$ when there exist orthonormal vector fields $E_{1}, E_{2}, \ldots, E_{r}$, along $\gamma$, such that:

$$
\begin{aligned}
& \dot{\gamma}=E_{1}, \nabla_{\dot{\gamma}} E_{1}=k_{1} E_{2}, \nabla_{\dot{\gamma}} E_{2}=-k_{1} E_{1}+k_{2} E_{3}, \ldots, \\
& \nabla_{\dot{\gamma}} E_{r-1}=-k_{r-2} E_{r-2}+k_{r-1} E_{r}, \nabla_{\dot{\gamma}} E_{r}=-k_{r-1} E_{r-1}
\end{aligned}
$$

where $k_{1}, k_{2}, \ldots, k_{r-1}$ are positive $C^{\infty}$ functions of $s . k_{j}$ is called the $j$-th curvature of $\gamma$.

So, for example, a geodesic is a Frenet curve of order 1 ; a circle is a Frenet curve of order 2 with $k_{1}$ a constant; a helix of order $r$ is a Frenet curve of order $r$, such that $k_{1}, k_{2}, \ldots k_{r-1}$ are constants. 
Proposition Let $M$ be a flat, 3-dimensional mass-symmetric 2-type integral submanifold of $S^{7} \subset E^{8}$. Then $M$ is locally the product of 3 curves. Any one of these curves is a helix of order 4 , or a circle in $E^{8}$.

Proof. According to Lemma 2, the Weingarten maps (3.1) of $M$ have constant components and $w_{i}^{j}=0, i, j,=1,2,3$. Thus, by using the GaussWeingarten formulas we have

$$
\begin{aligned}
& \bar{\nabla}_{X_{1}} X_{1}=\lambda_{1} \xi_{1}-x, \quad \bar{\nabla}_{X_{2}} X_{1}=\lambda \xi_{2}, \quad \bar{\nabla}_{X_{3}} X_{1}=\lambda \xi_{3} \\
& \bar{\nabla}_{X_{2}} X_{2}=\lambda \xi_{1}+a \xi_{2}-x, \quad \bar{\nabla}_{X_{3}} X_{2}=c \xi_{3}, \quad \bar{\nabla}_{X_{i}} x=X_{i} \\
& \bar{\nabla}_{X_{3}} X_{3}=\lambda \xi_{1}+c \xi_{2}+d \xi_{3}-x, \quad \bar{\nabla}_{X_{1}} \xi_{1}=-\lambda_{1} X_{1}+\xi \\
& \bar{\nabla}_{X_{2}} \xi_{1}=-\lambda X_{2}, \quad \bar{\nabla}_{X_{3}} \xi_{1}=-\lambda X_{3}, \quad \bar{\nabla}_{X_{2}} \xi_{2}=-\lambda X_{1}-a X_{2}+\xi \\
& \bar{\nabla}_{X_{3}} \xi_{2}=-c X_{3}, \quad \bar{\nabla}_{X_{3}} \xi_{3}=-\lambda X_{1}-c X_{2}-d X_{3}+\xi, \quad \bar{\nabla}_{X_{i}} \xi=-\xi_{i} .
\end{aligned}
$$

Let $X_{1}=E_{1}$. From (4.17) we obtain

$$
\begin{aligned}
& \bar{\nabla}_{E_{1}} E_{1}=\lambda_{1} \xi_{1}-x=k_{1} E_{2}, \text { where } k_{1}=\sqrt{1+\lambda^{2}}, E_{2}=\frac{1}{k_{1}}\left(\lambda \xi_{1}-x\right), \\
& \bar{\nabla}_{E_{1}} E_{2}=-k_{1} X_{2}+\frac{\lambda_{1}}{k_{1}} \xi=-k_{1} E_{1}+k_{2} E_{3}, \quad k_{2}=\frac{\lambda_{1}}{k_{1}} \text { and } E_{3}=\xi, \\
& \bar{\nabla}_{E_{1}} E_{3}=-\xi_{1}=-k_{2} E_{2}+k_{3} E_{4}, k_{3}=\frac{1}{k_{1}} \text { and } E_{4}=-\frac{1}{k_{1}}\left(\xi_{1}+\lambda_{1} x\right), \\
& \bar{\nabla}_{E_{1}} E_{4}=-k_{3} E_{3} .
\end{aligned}
$$

Thus $k_{4}=0$ and an $X_{1}$-curve of $M$ is a helix of order 4 in $E^{8}$. If $\lambda_{1}=0$, we have $k_{2}=0$ and the $X_{1}$-curve is a circle.

Now we put $X_{2}=E_{1}$. From (4.17) we obtain

$$
\begin{aligned}
& \bar{\nabla}_{E_{1}} E_{1}=\lambda \xi_{1}+a \xi_{2}-x=k_{1} E_{2}, \quad k_{1}=\sqrt{\lambda^{2}+a^{2}+1}, \\
& E_{2}=\frac{1}{k_{1}}\left(\lambda \xi_{1}+a \xi_{2}-x\right) . \\
& \bar{\nabla}_{E_{1}} E_{2}=\frac{1}{k_{1}}\left(-\lambda a X_{1}-k_{1}^{2} X_{2}+a \xi\right)=-k_{1} E_{1}+k_{2} E_{3}, \\
& k_{2}=\frac{\varepsilon a \sqrt{\lambda^{2}+1}}{k_{1}}, E_{3}=\frac{\varepsilon}{\sqrt{\lambda^{2}+1}}\left(-\lambda X_{1}+\xi\right), \\
& \text { where } \varepsilon= \pm 1 \text { according as } a>0 \text { or } a<0 .
\end{aligned}
$$




$$
\begin{gathered}
\bar{\nabla}_{E_{1}} E_{3}=-\varepsilon \sqrt{\lambda^{2}+1} \xi_{2}=-k_{2} E_{2}+k_{3} E_{4}, \quad k_{3}=\varepsilon \varepsilon_{1} \frac{1+\lambda^{2}}{k_{1}} \\
E_{4}=\frac{\varepsilon_{1}}{k_{1} \sqrt{\lambda^{2}+1}}\left(\lambda a \xi_{1}-\left(\lambda^{2}+1\right) \xi_{2}-a x\right) \\
\text { where } \varepsilon_{1}= \pm 1 \text { according as } \varepsilon= \pm 1 \\
\bar{\nabla}_{E_{1}} E_{4}=-k_{3} E_{3} .
\end{gathered}
$$

Thus $k_{4}=0$ and an $X_{2}$-curve of $M$ is a helix of order 4 in $E^{8}$. If $a=0$, we have $k_{2}=0$ and the curve is a circle.

Let now $X_{3}=E_{1}$. In the same manner, from (4.17) we have

$$
\begin{gathered}
\bar{\nabla}_{E_{1}} E_{1}=\lambda \xi_{1}+c \xi_{2}+d \xi_{3}-x=k_{1} E_{2}, \quad k_{1}=\sqrt{\lambda^{2}+c^{2}+d^{2}+1} \\
E_{2}=\frac{1}{k_{1}}\left(\lambda \xi_{1}+c \xi_{2}+d \xi_{3}-x\right) . \\
\bar{\nabla}_{E_{1}} E_{2}=\frac{1}{k}\left(-\lambda d X_{1}-c d X_{2}-k_{1}^{2} X_{3}+d \xi\right)=-k_{1} E_{1}+k_{2} E_{3}, \\
k_{2}=\frac{\varepsilon d}{k_{1}} \sqrt{\lambda^{2}+c^{2}+1}, \\
E_{3}=\frac{\varepsilon}{\sqrt{\lambda^{2}+c^{2}+1}}\left(-\lambda X_{1}-c X_{2}+\xi\right), \\
\text { where } \varepsilon= \pm 1 \text { according as } d>0 \text { or } d<0 . \\
\bar{\nabla}_{E_{1}} E_{3}=-\varepsilon \sqrt{\lambda^{2}+c^{2}+1} \xi_{3}=-k_{2} E_{2}+k_{3} E_{4}, \\
k_{3}=\frac{\varepsilon \varepsilon_{1}}{k_{1}}\left(\lambda^{2}+c^{2}+1\right) \\
E_{4}=\frac{\varepsilon_{1}}{k_{1} \sqrt{\lambda^{2}+c^{2}+1}}\left(\lambda d \xi_{1}+c d \xi_{2}-\left(\lambda^{2}+c^{2}+1\right) \xi_{3}-d x\right), \\
\quad \text { where } \varepsilon_{1}= \pm 1 \text { according as } \varepsilon= \pm 1 . \\
\bar{\nabla}_{E_{1} E_{4}=}-k_{3} E_{3} .
\end{gathered}
$$

Thus an $X_{3}$-curve is a helix of order 4 . If $d=0$, the curve is a circle.

\section{References}

[1] Baikoussis C. and Blair D.E., 2-type integral surfaces in $S^{5}(1)$. Tokyo J. Math. 14 (1991), 277-288.

[2] Baikoussis C., Blair D.E. and Koufogiorgos T., Integral submanifolds of Sasakian space forms $\bar{M}^{7}(k)$. Results in Mathematics 27 (1995), 207-226.

[3] Baikoussis C., Defever F. and Koufogiorgos T., Coordinate finite type integral surfaces in $S^{5}$. Algebras, Groups and Geom. 10 (1993), 227-239. 
[4] Blair D.E., Contact manifolds in Riemannian geometry. Lecture Notes in Mathematics 509 (1976), Springer-Verlag.

[5] Chen B.-Y., On the total curvature of immersed manifolds. VI: Submanifolds of finite type and their applications, Bull. Inst. Math. Acad. Sinica 11 (1983), 309-328.

[6] Chen B.-Y., Total Mean Curvature and Submanifolds of Finite Type. World Scientific, 1984.

[7] Chen B.-Y., Some open problems and conjectures on submanifolds of finite type. Soochow J. Math. 17 (1991), 169-188.

[8] Ogiue K., Notes in Differential Geometry. Universidad de Granada, Lecture Notes by O.J. Garay, 1985.

[ 9 ] Sasaki S., A characterization of contact transformations. Tôhoku Math. J. 16 (1964), 285-290.

[10] Takahashi T., Minimal immersions of Reimannian manifolds. J. Math. Soc. Japan 18 (1966), 380-385.

Christos Baikoussis

Department of Mathematics

University of Ioannina

Ioannina 45110

Greece

E-mail: cbaikou@cc.uoi.gr

David E. Blair

Department of Mathematics

Michigan State University

East Lansing, MI 48824

U.S.A.

E-mail: blair@math.msu.edu 